\title{
热处理工艺对 $\mathrm{Ti}_{2} \mathrm{AINb}$ 惯性摩擦焊 接头组织及性能的影响*
}

\author{
周军 梁武 乌彦全 张春波 \\ (哈尔滨焊接研究院有限公司 哈尔滨 150028)
}

\begin{abstract}
摘要: 针对 $\mathrm{Ti}_{2} \mathrm{AINb}$ 合金惯性摩擦焊接接头开展了热处理工艺试验研究, 分析了不同热处理工艺对焊接接头组织及力学性能 的影响。研究表明, 固溶态母材由 $\alpha_{2} 、 \mathrm{~B} 2$ 和 $\mathrm{O}$ 相构成, $\alpha_{2}$ 相呈球状或块状分布于 B2 相晶界上, $\mathrm{O}$ 相呈棒状平行或交叉分 布于 $\mathrm{B} 2$ 相晶粒内部; 固溶 + 单时效态合金母材由 $\mathrm{B} 2+\mathrm{O}$ 两相组成。焊态下的接头焊缝区及热力影响区基本为 $\mathrm{B} 2$ 相组织, 双 时效处理后大部分 $\mathrm{B} 2$ 相转变成晶界网状 $\mathrm{O}$ 相十晶内针状及块状 $\mathrm{O}$ 相组织, 单时效处理的接头焊缝区及热力影响区晶界基本 无 $\mathrm{O}$ 相组织存在, $\mathrm{B} 2$ 相晶内转变为细密层片状 $\mathrm{B} 2+\mathrm{O}$ 相共析组织。三种热处理状态的接头各区域显微硬度分布基本一致, 焊后双时效提高了 $\mathrm{O}$ 相组织的析出而起到强化作用并提高了接头的显微硬度。焊前为固溶态十焊后双时效处理的接头室温抗 拉强度最高且为 $1193 \mathrm{MPa}$; 焊后双时效处理的接头室温拉伸断口以准解理断裂为主, 焊后单时效处理的接头室温拉伸断口 以解理断裂为主, 且解理小平面尺寸较大, 抗拉强度稍低。焊前为固溶态+焊后双时效处理的接头在 $650{ }^{\circ} \mathrm{C}$ 高温下的抗拉强 度依然最高且为 $986 \mathrm{MPa}$, 断口上能够观察到较浅的韧窝, 为沿晶的韧性断裂; 焊前为固溶态+时效处理的接头断口形貌基 本相同, 接头断裂形式以解理断裂为主, 并含有少量的准解理断口。
\end{abstract}

关键词: $\mathrm{Ti}_{2} \mathrm{AlNb}$; 惯性摩擦焊; 组织; 力学性能

中图分类号: TG456

\section{Effect of Heat Treatment on Microstructure and Properties of Inertial Friction Welded $\mathbf{T i}_{2}$ AlNb Joint}

\author{
ZHOU Jun LIANG Wu WU Yanquan ZHANG Chunbo
}

(Harbin Welding Institute Limited Company, Harbin 150028)

\begin{abstract}
Inertia friction welded $\mathrm{Ti}_{2} \mathrm{AlNb}$ alloy joints are subjected to heat treatment. The effects of different heat treatment processes on the microstructure and mechanical properties of welded joints are analyzed. The results indicate that base metal shows a typical microstructure comprised of $\alpha_{2}, \mathrm{~B} 2$ and $\mathrm{O}$ three phases. Base metal of aging treatment shows a typical microstructure comprised of $\mathrm{B} 2+\mathrm{O}$ phases. The microstructure of weld zone(WZ) and thermo-mechanically affected zone(TMAZ) of welded joints is main B2 phase. After double aging treatment, most B2 phase of WZ and TMAZ has transformed to network O phase in grain boundary and the needle with massive $\mathrm{O}$ phase within the grain. After aging treatment, $\mathrm{O}$ phase is not found in the grain boundary of WZ and TMAZ, and B2 phase transform into close and lamellar eutectoid structure of B2+O phase. The microhardness distribution of the joints in the three heat treatment states is basically the same in all areas. Double aging after welding improves the precipitation of $O$ phase and forms an precipitation strengthening, which improve the microhardness of joints. The tensile strength of welded joint of solid solution before welding and double aging treatment after welding is the highest and is $1193 \mathrm{MPa}$ at room temperature. Tensile fracture of double aging after welding is mainly quasi cleavage fracture, that the room temperature tensile fracture of the joints of single aging after welding is mainly cleavage fracture, which the size of cleavage plane is relatively large. The tensile strength of joints of solid solution before welding and double aging treatment after welding is still the highest and is $986 \mathrm{MPa}$ at $650{ }^{\circ} \mathrm{C}$ high temperature, and shallow dimples can be observed on the fracture that it is ductile fracture along grain boundary. However, the fracture morphology of joints of solid solution and aging treatment before welding is basically the same. The fracture form of the joints is mainly cleavage fracture and contains a small amount of quasi cleavage fracture.
\end{abstract}

Key words: $\mathrm{Ti}_{2} \mathrm{AlNb}$; inertia friction welded; microstructure; mechanical property

* 国家科技重大专项(2017ZX04004001, 2018ZX04010001)和重点研发省级 资金(GX18A035)资助项目。20190830 收到初稿, 20191126 收到修改稿 


\section{0 前言}

$\mathrm{Ti}_{2} \mathrm{AlNb}$ 金属间化合物是以 $\mathrm{O}$ 相为主相, $\mathrm{B} 2$ 相 和 $\alpha_{2}$ 相为辅相的一种新型轻质耐高温结构材料 ${ }^{[1]}$, 其中 $\alpha_{2}$ 相为有序密排六方的 D019 超点阵结构, 是 $\alpha-\mathrm{Ti}$ 结构的有序化结构 ${ }^{[2]}$, 而 $\mathrm{Nb}$ 原子取代了具有密 排六方超点阵结构的 $\alpha_{2}$ 相晶格节点上的原子后形成 的具有正交晶格结构的 $\mathrm{O}$ 相组织, $\mathrm{Ti}_{2} \mathrm{AlNb}$ 合金成 分通常在 Ti-(18-30)Al-(12.5-30)Nb 范围, 并含有少 量的 Mo、V、Ta 等合金元素, 因其具有低密度、 高比强度及良好的抗氧化、耐高温等优点 ${ }^{[3]}$, 已被 视为有助于通过结构减重实现航空发动机性能提升 的理想轻比重耐高温结构材料 ${ }^{[4]}$, 对降低航空发动 机重量, 提高推重比具有重要战略意义。

近年来, 国内外学者在研究 $\mathrm{TiAl} 、 \mathrm{Ti}_{3} \mathrm{Al}$ 等金属 间化合物焊接工艺基础上 ${ }^{[5-11]}$, 进一步开展了 Ti-Al 系合金中 $\mathrm{Ti}_{2} \mathrm{AlNb}$ 新型金属间化合物的熔化焊及摩 擦焊接工艺研究 ${ }^{[12-14]}$, 因摩擦焊接过程中材料是在 热塑性状态下实现的类锻态固相连接, 接头晶粒组织 细小, 其力学性能要远高于熔化焊方法。其中 CHEN 等 ${ }^{[15-16]}$ 研究 $\mathrm{Ti}_{2} \mathrm{AlNb}$ 合金线性摩擦焊接及焊后热处 理对焊接接头组织及性能影响中得到了室温抗拉强 度达到 $1136 \mathrm{MPa}$ 的线性摩擦焊接头, 贺建超等 ${ }^{[17]}$ 在研究 $\mathrm{Ti}_{2} \mathrm{AlNb}$ 合金的线性摩擦焊接头组织分布中 也得到了与 CHEN 相近的研究结果。周军等 ${ }^{[18]}$ 探索 性的研究了 $\mathrm{Ti}_{2} \mathrm{AlNb}$ 合金惯性摩擦焊接头的组织类 型及显微硬度分布。

本文在前期研究工作的基础上, 进一步开展了 不同热处理状态下的 $\mathrm{Ti}_{2} \mathrm{AlNb}$ 合金惯性摩擦焊接工 艺试验及焊后热处理工艺试验研究, 分析总结了焊 接及热处理工艺参数对 $\mathrm{Ti}_{2} \mathrm{AlNb}$ 合金惯性摩擦焊接 接头组织及力学性能的影响。

\section{1 试验设备及材料}

\section{1 试验设备}

试验设备采用哈尔滨焊接研究院有限公司自主 研发设计的型号为 HWI-IFW-130 型轴/径向惯性摩 擦焊机, 设备最大轴向焊接力为 $1300 \mathrm{kN}$, 主轴最 高转速为 $1000 \mathrm{r} / \mathrm{min}$, 自动焊接过程采用转速控制 方式, 设备如图 1 所示。

\section{2 试验材料}

试验材料采用 $\mathrm{Ti}-20 \mathrm{Al}-25 \mathrm{Nb}$ 型 $\mathrm{Ti}_{2} \mathrm{AlNb}$ 合金锻 件, 工件端面外径尺寸为 $100 \mathrm{~mm}$, 壁厚为 $15 \mathrm{~mm}$, 供货状态为锻造+固溶热处理。图 $2 a$ 和 $2 b$ 分别为

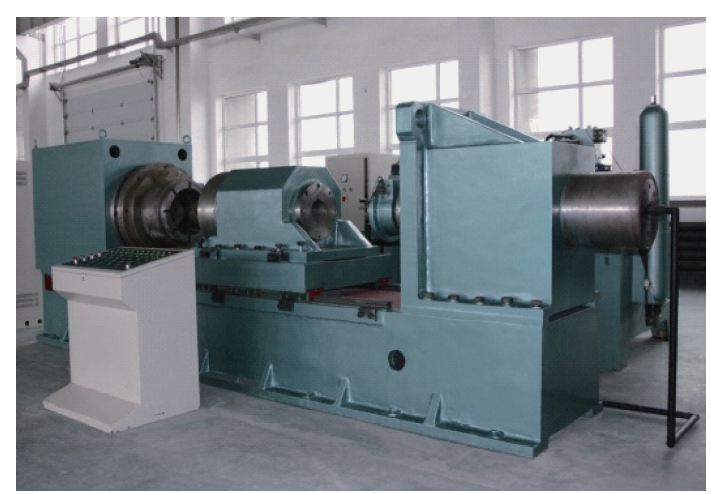

图 1 HWI-IFW-130 型轴/径向惯性摩擦焊机

$\mathrm{Ti}_{2} \mathrm{AlNb}$ 合金固溶态及固溶 $+990{ }^{\circ} \mathrm{C} \times 2 \mathrm{~h}$ 时效态下母 材显微组织, 经 X 射线衍射分析可知, 如图 3 所示, 固溶态下的 $\mathrm{Ti}_{2} \mathrm{AlNb}$ 合金母材组织由 $\alpha_{2}$ 相、 $\mathrm{B} 2 / \beta$ 相 和 $\mathrm{O}$ 相组成, 晶粒尺寸约为 $40 \mu \mathrm{m}$ 。 $\alpha_{2}$ 相呈近球状 断续分布于原 $\mathrm{B} 2 / \beta$ 等轴晶晶界, 直径约为 $5 \mu \mathrm{m} ; \mathrm{O}$ 相呈棒状平行或交错弥散分布于 $B 2 / \beta$ 基体上。固溶 $+990{ }^{\circ} \mathrm{C} \times 2 \mathrm{~h}$ 时效态下的母材显微组织转变为 $B 2+\mathrm{O}$ 相的两相组织, $\alpha_{2}$ 相消失, $\mathrm{O}$ 相组织在晶界 呈近球状或块状分布, 在 B2 相晶内呈针状分布, 在高倍镜下观察到 B2 相组织疑似分解。

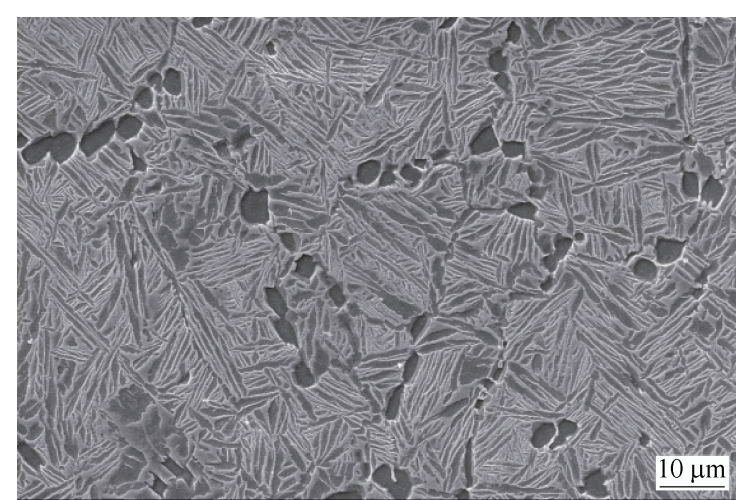

(a) 固溶态

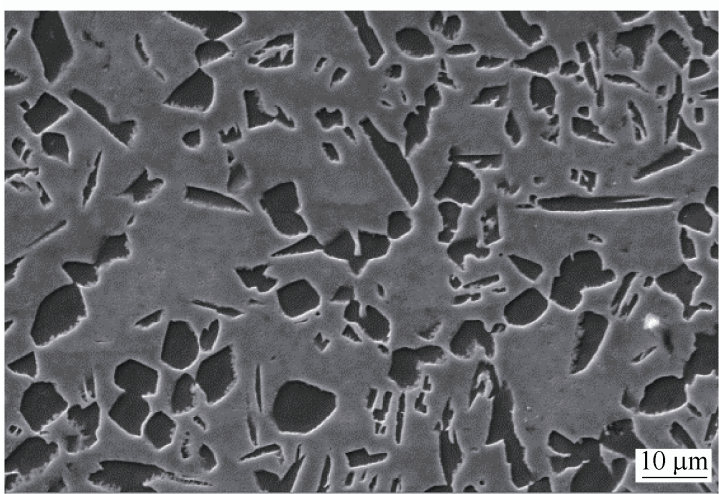

(b) 固溶 $+990^{\circ} \mathrm{C} \times 2 \mathrm{~h}$ 空冷时效

图 $2 \mathrm{Ti}_{2} \mathrm{AlNb}$ 合金母材显微组织

\section{3 焊接及热处理工艺}

为研究不同热处理制度对 $\mathrm{Ti}_{2} \mathrm{AlNb}$ 合金惯性摩 擦焊接头组织及性能的影响, 采用了多组热处理工 


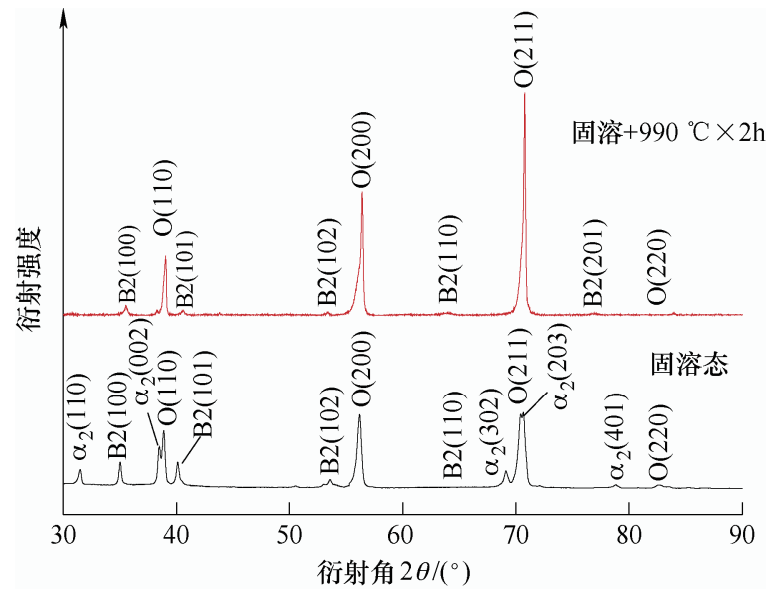

图 $3 \mathrm{Ti}_{2} \mathrm{AlNb}$ 合金母材 $\mathrm{X}$ 射线衍射分析

艺参数, 如表 1 所示, 并应用图 1 所示的惯性摩 擦焊机完成了 $\mathrm{Ti}_{2} \mathrm{AlNb}$ 合金的焊接, 焊接工艺参数 如表 2 所示。两组接头焊接过程稳定, 其焊接接 头外观形貌如图 4 所示, 其中\#1 焊接飞边形貌均 匀, 且内、外飞边均无未出现明显的宏观裂纹缺 陷; \#2 焊接飞边形貌不均, 在焊接飞边外表面出 现垂直于焊缝的轴向裂纹缺陷, 左下角为飞边局 部形貌。图 5 为 $\mathrm{Ti}_{2} \mathrm{AlNb}$ 合金惯性摩擦焊接头焊缝 典型宏观形貌图, 接头飞边成形良好, 且能够明 显区分焊缝区、热力影响区及热影响区。焊后采 用机加工方法去除\#1 和\#2 焊接接头内外飞边, 并 对接头进行渗透探伤检测, 焊接接头均未发现周 向或轴向分布裂纹缺陷, 说明接头上的宏观裂纹 缺陷仅存在焊接飞边处, 且裂纹未扩展至焊缝及 母材基体，焊缝质量良好。

表 1 热处理工艺参数

\begin{tabular}{ccc}
\hline 序号 & 焊前 & 焊后 \\
\hline$\# 1$ & 固溶态 & $990{ }^{\circ} \mathrm{C} \times 2 \mathrm{~h}$ 空冷 $+790{ }^{\circ} \mathrm{C} \times 24 \mathrm{~h}$ 空冷 \\
$\# 2-1$ & 固溶态 $+990{ }^{\circ} \mathrm{C} \times 2 \mathrm{~h}$ 空冷 & $790{ }^{\circ} \mathrm{C} \times 24 \mathrm{~h}$ 空冷 \\
$\# 2-2$ & & $990{ }^{\circ} \mathrm{C} \times 2 \mathrm{~h}$ 空冷 $+7900^{\circ} \mathrm{C} \times 24 \mathrm{~h}$ 空冷 \\
\hline
\end{tabular}

表 2 焊接工艺参数

\begin{tabular}{cccc}
\hline $\begin{array}{c}\text { 初始转速/ } \\
(\mathrm{r} / \mathrm{min})\end{array}$ & $\begin{array}{c}\text { 转动惯量/ } \\
\left(\mathrm{kg} \cdot \mathrm{m}^{2}\right)\end{array}$ & $\begin{array}{c}\text { 摩擦压力/ } \\
\mathrm{MPa}\end{array}$ & $\begin{array}{c}\text { 顶锻压力/ } \\
\mathrm{MPa}\end{array}$ \\
\hline 700 & 90 & 30 & 60 \\
\hline
\end{tabular}

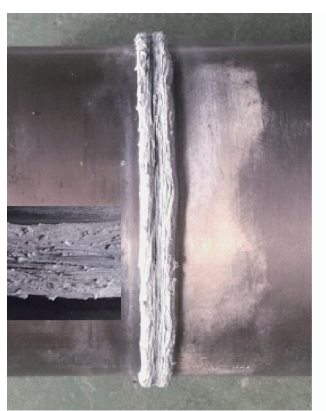

(a) 1 号焊接接头

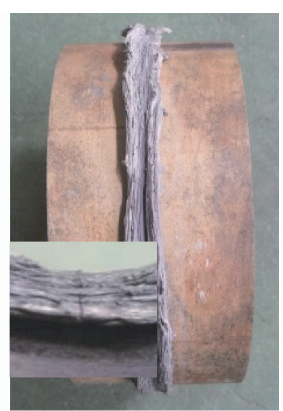

(b) 2 号焊接接头
图 4 焊接接头飞边形貌

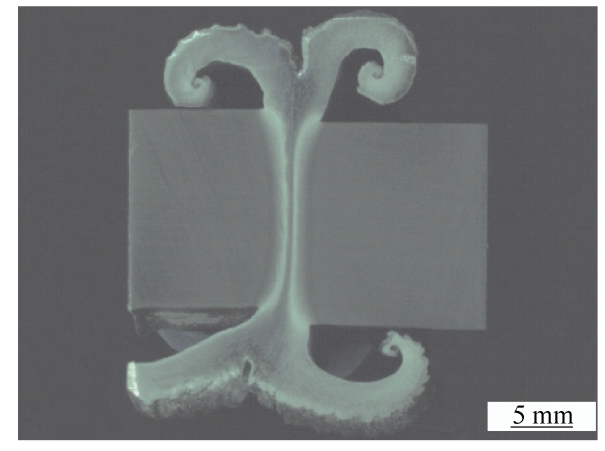

图 $5 \mathrm{Ti}_{2} \mathrm{AlNb}$ 接头焊缝宏观形貌

\section{2 试验结果及分析}

\section{1 焊接接头显微组织分析}

\subsection{1 焊缝区}

$\mathrm{Ti}_{2} \mathrm{AlNb}$ 作为由 $\mathrm{O}$ 相、 $\alpha_{2}$ 相和 $\mathrm{B} 2 / \beta$ 相组成的三 相合金, 接头焊缝区融合良好, 两侧金属形成共有 晶粒, 融合界面消失。图 4 为\#1 焊接接头焊缝区显 微组织, 焊接过程中, 由于工件端面间的摩擦作用 使得界面温度已处于 B2 相的单相区内, 致使大量 的棒状 $O$ 相组织和等轴状 $\alpha_{2}$ 相组织全部转变成了 B2 相组织, 冷却过程中, 形成了完全由亚稳态 B2 相等轴晶十沿晶析出的少量断续网状 $\mathrm{O}$ 相组成的焊 缝区组织, 但未观察到 $\alpha_{2}$ 相组织存在, B2 相晶粒直 径约为 $20 \mu \mathrm{m}$, 如图 6a 所示。焊接接头经过 $990{ }^{\circ} \mathrm{C} \times$ $2 \mathrm{~h}$ 空冷 $+790{ }^{\circ} \mathrm{C} \times 24 \mathrm{~h}$ 空冷双时效处理后, $\mathrm{O}$ 相组 织沿等轴 B2 相晶界呈网状析出, 在 B2 相晶粒内部 呈块状及针状析出, 针状 $\mathrm{O}$ 相组织基本呈平行分布 且有少量出现交叉状态, 其数量远低于固溶态下母 材中的 $\mathrm{O}$ 相组织, 尺寸也明显减小, 如图 $6 \mathrm{~b}$ 所示。

图 7 为焊前固溶+时效状态的\#2 接头焊缝区在 焊态及不同焊后热处理态下的显微组织形貌。图 7a 与图 6a 组织类型及形貌基本相同, 在焊态下焊缝区 均由 B2 相等轴晶十晶界断续网状 $\mathrm{O}$ 相构成, 晶粒直 径相当。经过不同焊后时效处理的焊缝区组织具有 明显区别, 焊后单时效处理的\#2-1 接头焊缝区原始 等轴状 B2 相晶粒整体发生共析转变, 形成了细密

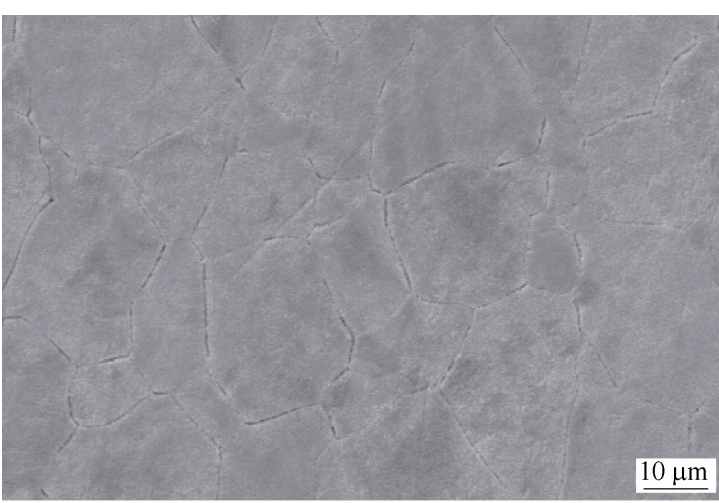

(a) 焊态 


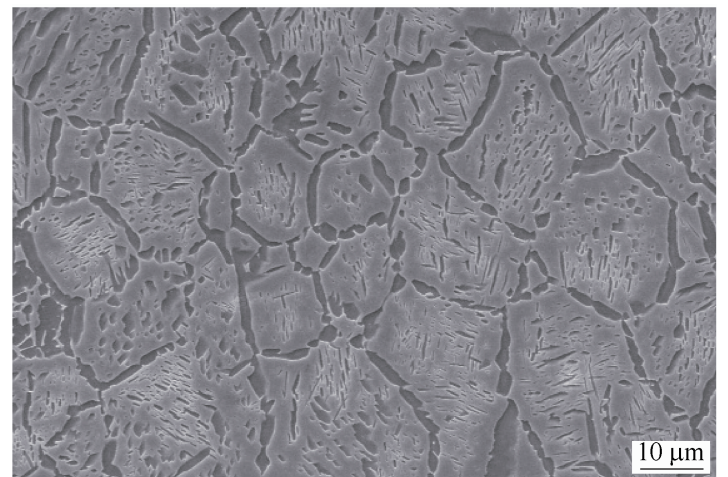

(b) 焊后热处理态

图 6 \#1 焊接接头焊缝区显微组织

层片状的 $\mathrm{B} 2+\mathrm{O}$ 相共析组织, B2 相晶界断续网状 $\mathrm{O}$ 相组织未见明显变化, 如图 $7 \mathrm{~b}$ 所示。经过焊后双时 效处理的\#2-2 接头焊缝区组织类型及形貌与\#1 基 本相同, 但\#2-2 接头焊缝区的 B2 相晶粒内析出的 板条状 $\mathrm{O}$ 相组织数量更多, 板条厚度更小, 仅有约 $1 \mu \mathrm{m}$, 且沿 B2 相晶粒边界析出的链状 $\mathrm{O}$ 相组织尺 寸略微小于\#1 接头, 如图 7c 所示。

\#1 和\#2-2 接头焊后时效温度较高, 热能驱动力 充足, 金属原子可实现快速、长距离扩散, $\mathrm{O}$ 相可 充分在 $\mathrm{B} 2$ 相晶界及晶内形核积聚长大, 在晶界及 晶内形成大尺寸的 $\mathrm{O}$ 相组织。但\#2-1 接头焊后时效 温度较低, 热能驱动力不足, 形核速度尚可, 积聚 长大速度较慢, 最终在 $\mathrm{B} 2$ 相晶粒内部形成细密层 片状的 $\mathrm{B} 2+\mathrm{O}$ 相共析组织。

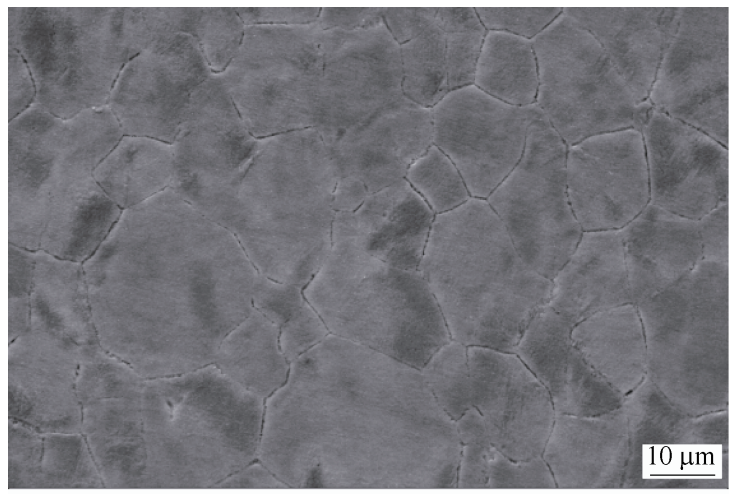

(a) 焊态

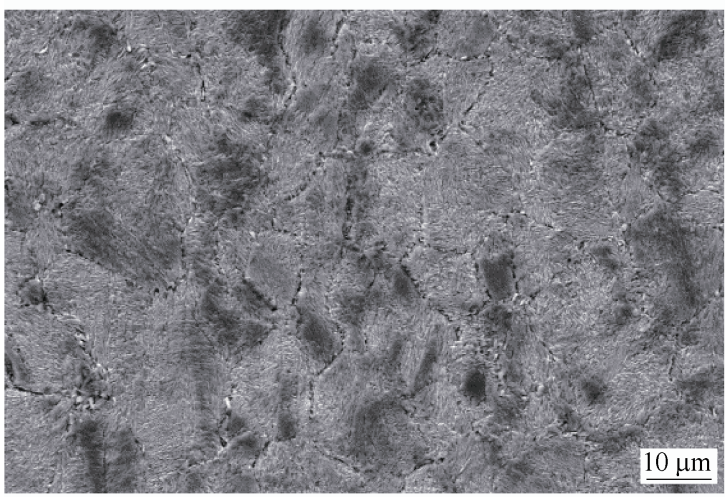

(b) $2-1$ 号焊后热处理态

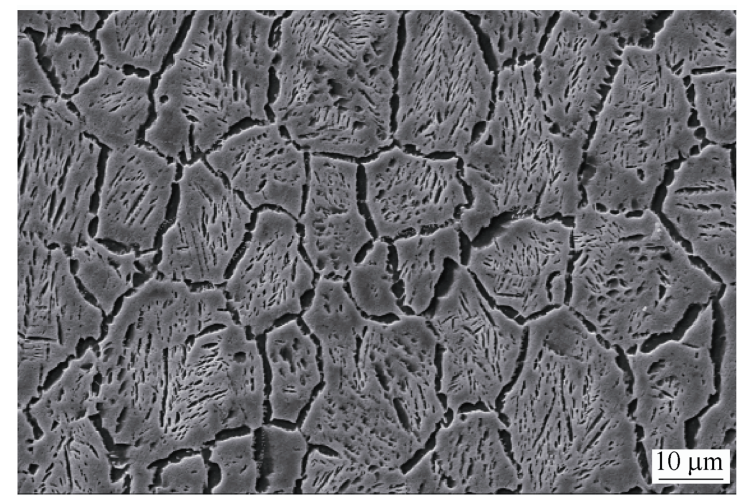

(c) 2-2号焊后热处理态

图 7 \#2 焊接接头焊缝区显微组织

\subsection{2 热力影响区}

图 $8 a$ 和图 $9 b$ 分别为 $\# 1$ 和 $\# 2$ 接头焊态下的热力 影响区显微组织形貌, 基本为 B2 相组织。由于受 轴向力及热的作用, 使 B2 相晶粒发生了变形和动 态再结晶, 沿流变方向被拉长。由于温度较高, 晶 粒长大速度较快, $\mathrm{B} 2$ 相晶粒变形量不大, 动态再结 晶过程不充分, 导致晶粒比较粗大。 $\mathrm{B} 2$ 相晶粒基体 上存在较多被拉长的凹坑, 该凹坑处原来应是 $\alpha_{2}$ 相 或大块状的 $\mathrm{O}$ 相组织, 由于溶解后扩散不充分, 化 学成分未达到 B2 相平衡成分, 耐蚀性较差形成模 糊的凹坑。

$\# 1$ 接头热力影响区经过双时效处理后, 如图 $8 b$

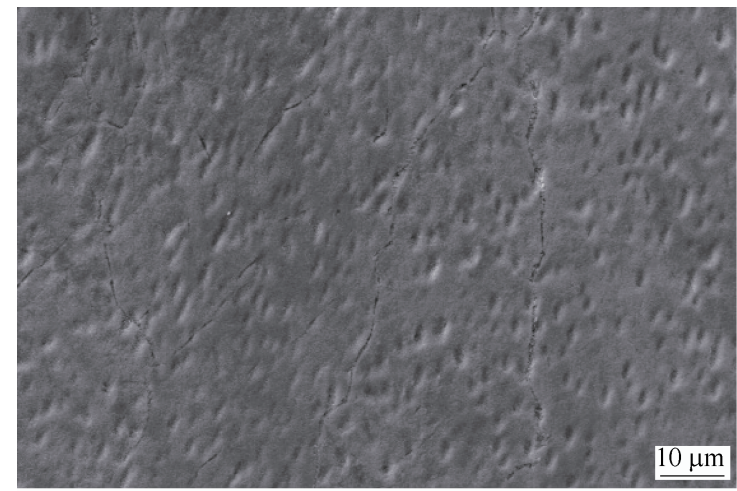

(a) 焊态

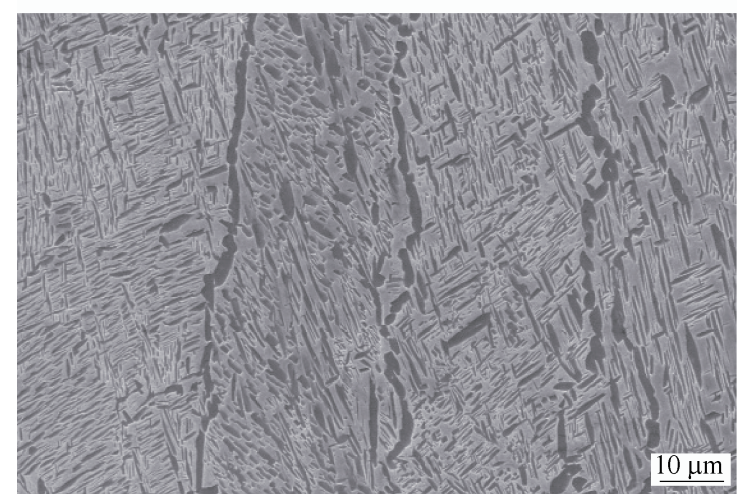

(b) 焊后热处理态

图 8 \#1 焊接接头热力影响显微组织 


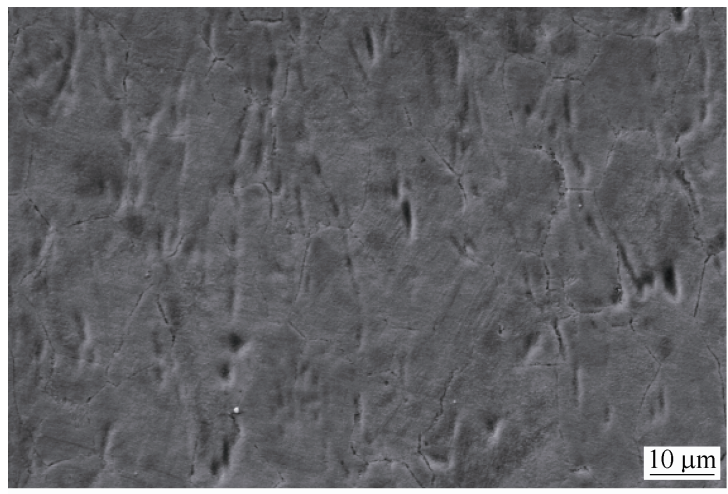

(a) 焊态

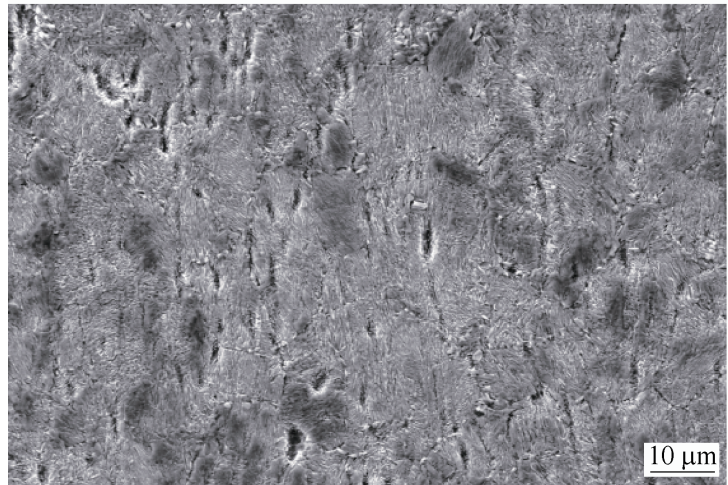

(b) \#2-1 焊后热处理态

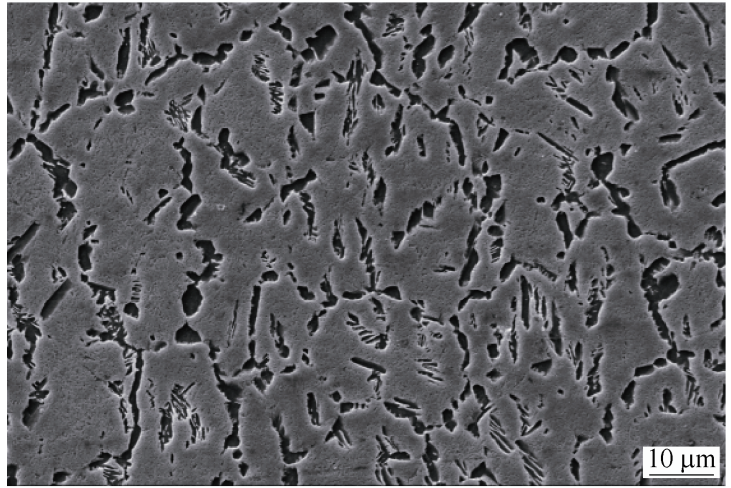

(c) \#2-2 焊后热处理态

图 9 \#2 焊接接头热力影响区显微组织

所示, 原始 B2 相晶粒形态依然保持焊态, 沿着细 长、粗大的 $\mathrm{B} 2$ 相晶粒边界析出了链状 $\mathrm{O}$ 相组织。 在 $\mathrm{B} 2$ 相晶粒内部, 细小棒状 $\mathrm{O}$ 相组织呈平行或 部分交叉分布于 $\mathrm{B} 2$ 相基体上。\#2-2 接头热力影响 区晶粒基本呈拉长状态, B2 相晶界上的 $\mathrm{O}$ 相组织 呈不连续的块状, 其数量远高于\# 1 接头 $\mathrm{B} 2$ 相晶 界上的 $\mathrm{O}$ 相组织, 所以, $\mathrm{B} 2$ 相基体内 $\mathrm{Nb}$ 含量较 高, 在低温时效过程中 B2 相组织分解不明显, 组 织较细不易腐蚀。经过焊后双时效处理的\#2-2 和 \#1 焊接接头热力影响区凹坑已消失。经过焊后单 时效的\#2-1 接头热力影响区 B2 相晶粒内已发生 共析转变, 转变成较细密的层片状 $\mathrm{B} 2+\mathrm{O}$ 相组织, 晶界末见沿晶分布的 $\mathrm{O}$ 相组织, 焊态时呈现的长 条状凹坑依然可见, 凹坑内多为 $\mathrm{O}$ 相, 个别可见 残余 $\alpha_{2}$ 相组织。

\section{2 焊接接头力学性能分析}

\subsection{1 显微硬度}

图 10 为不同焊后热处理状态下的 $\mathrm{Ti}_{2} \mathrm{AlNb}$ 合金 惯性摩擦焊接头显微硬度变化曲线, 该曲线显示出 了不同热处理状态下的焊接接头上从焊缝到母材显 微硬度的变化趋势。从图 10 中可以清晰地观察到, 三种热处理制度的接头焊缝区整体显微硬度略低于 其他区域, 但显微硬度差值变化不大, 与热力影响 区、热影响区及母材基本保持一致。其中\#2-2 号接 头显微硬度最低, \#1 号接头显微硬度要低于\#2-2 接 头, 但与\#2-2 号接头显微硬度相差较小, 而\#2-1 号 接头各区域整体显微硬度最低。通常 $\mathrm{Ti}_{2} \mathrm{AlNb}$ 合金 中 $\mathrm{O}$ 相组织对提高显微硬度起到重要作用 ${ }^{[19]}$, 晶界 $\alpha_{2}$ 相的尺寸及数量对改变各区域显微硬度也将起到 一定作用, 其中\#1 号与\#2-2 号接头的焊缝区及热力 影响区中 $\mathrm{B} 2$ 相晶粒内部的块状 $\mathrm{O}$ 相组织及晶界上 的板条状 $O$ 相组织数量远多于\#2-1 号接头的焊缝区 及热力影响区, 显著提高了该区域的显微硬度值。

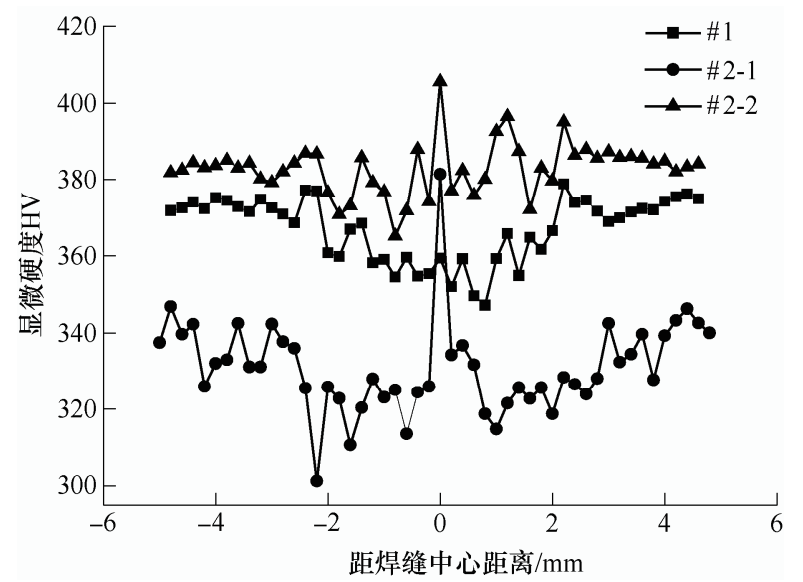

图 10 接头显微硬度变化曲线

\subsection{2 室温拉伸性能}

图 11 为 $\mathrm{Ti}_{2} \mathrm{AlNb}$ 合金焊接接头室温拉伸断后试 样, 室温拉伸下的\#1 和\#2-2 焊接接头拉伸试样均断 裂于母材区，焊接接头表现出了高于母材的强度性 能, 而\#2-1 焊接接头拉伸试样断裂于近焊缝区, 三 种接头断裂位置均无明显缩颈现象, 塑形相对较差。

$\# 1$

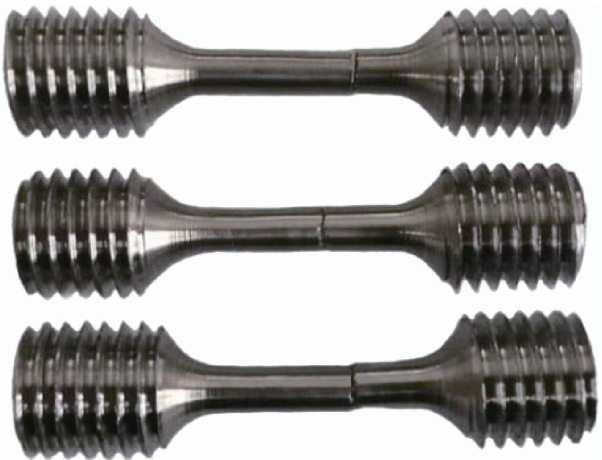

图 $11 \mathrm{Ti}_{2} \mathrm{AlNb}$ 合金焊接接头室温拉伸断后试样 
检测结果显示, 三种接头的抗拉强度值分别为 $\sigma_{\mathrm{b} \# 1}=$ $1193 \mathrm{MPa} 、 \sigma_{\mathrm{b} \# 2-1}=1100 \mathrm{MPa} 、 \sigma_{\mathrm{b} \# 2-2}=1170 \mathrm{MPa}$ 。其 中\#1 号焊接接头抗拉强度最高为 $1193 \mathrm{MPa}$, 表现出 了较好的强度性能, \#2-2 号焊接接头抗拉强度相对 次之, 但要高于具有相同焊前热处理状态的\#2-1 号 接头。\#1 焊接接头抗拉强度仅高于\#2-2 接头 $23 \mathrm{MPa}$, 差距相对较小, 但比\#2-1 接头抗拉强度高出 $93 \mathrm{MPa}$ 。

针对拉伸试验后的断口形貌开展了进一步分析 研究, 如图 12 所示。从图中可以观察到, 三种热处 理制度下的接头断口形貌特征具有一定的差别, 其 中\#1 接头为准解理断裂, \#2-1 接头以解理断裂为 主, 并含有少量准解理断口, 且解理断裂小平面尺 寸较大, 断裂面可能受焊接热循环影响较多, 焊后 单时效处理对断裂方式可能有一定影响。\#2-2 接头 断口为准解理断口, 局部可见沿球形析出相(能谱分 析球形析出物为 $\mathrm{O}$ 相)界面开裂。

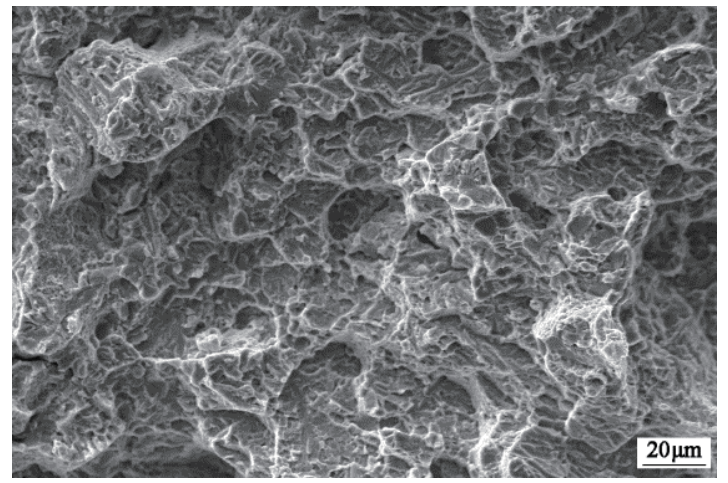

(a)\#1

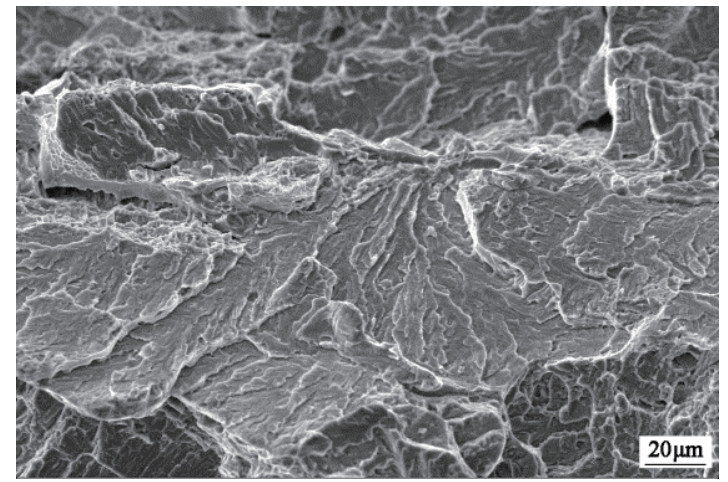

(b)\#2-1

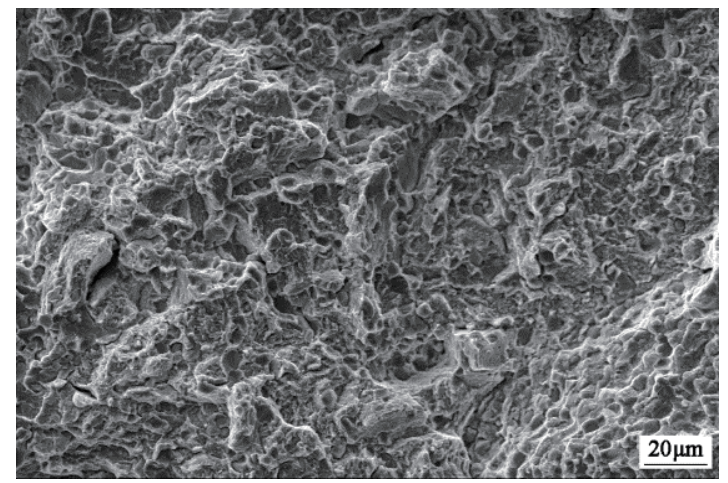

(c)\#2-2

图 $12 \mathrm{Ti}_{2} \mathrm{AlNb}$ 合金焊接接头室温拉伸断口形貌

\subsection{3 高温拉伸性能}

图 13 为 $\mathrm{Ti}_{2} \mathrm{AlNb}$ 合金焊接接头在 $650{ }^{\circ} \mathrm{C}$ 高温 条件下的拉伸断后试样。从图 13 中可以清晰地观 察到, $650{ }^{\circ} \mathrm{C}$ 高温条件下的拉伸试样也均断裂于母 材区，但断裂形式发生了一定的变化，其中\#1 接 头在 $650{ }^{\circ} \mathrm{C}$ 高温下依然表现出了一种脆性断裂特 征，断裂位置轴向平直几乎无明显缩颈现象，抗拉 强度更是达到了 $986 \mathrm{MPa}$ ，仅比室温下的\#1 号接头 抗拉强度低 $207 \mathrm{MPa}$ 。\#2-1 和\#2-2 号接头在 $650{ }^{\circ} \mathrm{C}$ 高温下却表现出了良好的塑性特征, 断裂位置出现 了明显的缩颈现象，高温条件下的抗拉强度值分别 为 $760 \mathrm{MPa}$ 和 $738 \mathrm{MPa}$, 远低于相同热处理制度下 的室温接头抗拉强度, 甚至比相同高温条件下的\#1 接头抗拉强度值都低了 $220 \mathrm{MPa}$ 以上, 拉伸性能相 对较差。

\#1

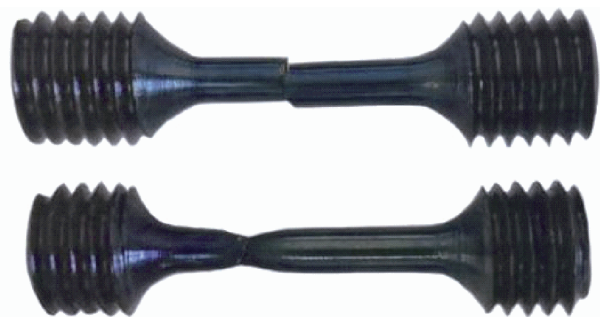

\#2-2

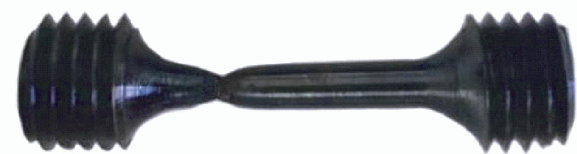

图 $13 \mathrm{Ti}_{2} \mathrm{AlNb}$ 合金焊接接头 $650{ }^{\circ} \mathrm{C}$ 高温拉伸断后试样

图 14 为三种热处理状态的下高温拉伸接头断 口微观形貌。其中\#2-1 和\#2-2 号接头断口形貌基 本相同, 接头断裂形式以解理断裂为主, 并含有 少量的准解理断口, 且解理断裂小平面尺寸较大, 解理断裂小平面尺寸与晶粒尺寸相当, 而\#1 号接 头为沿晶韧性断裂，断口上能够观察到较浅的韧 窝特征。

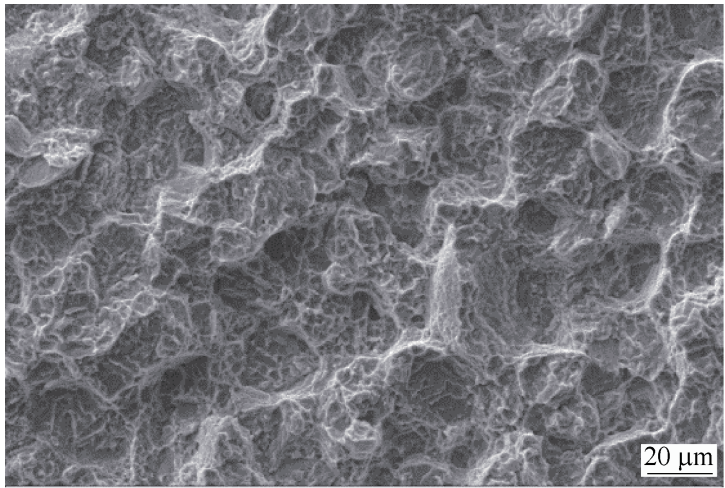

(a) \#1 


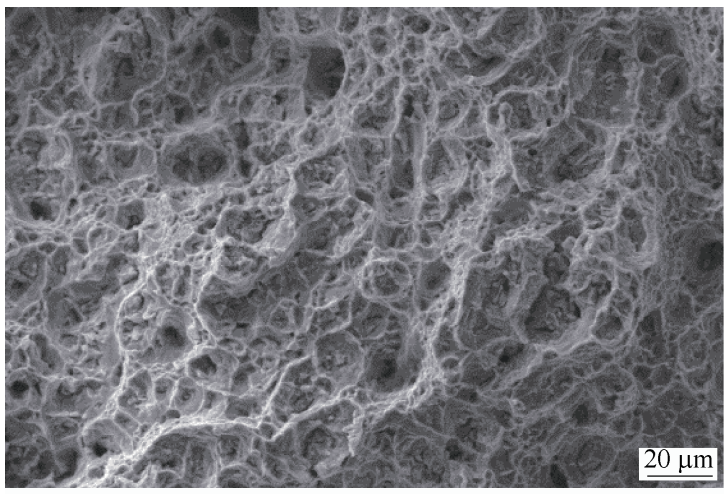

(b) \#2-1

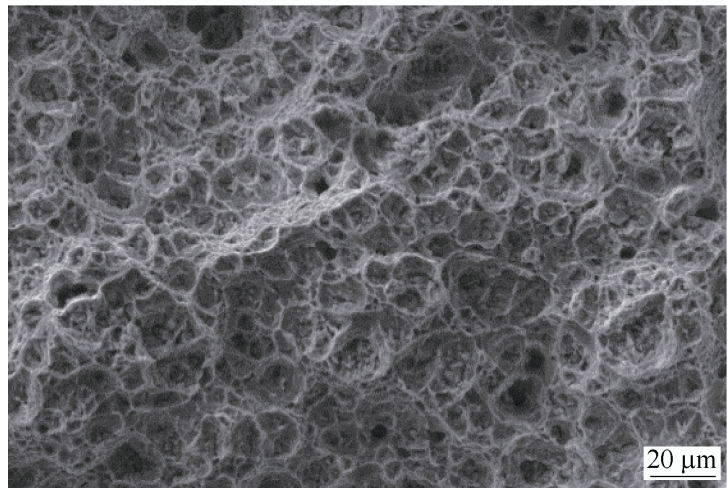

(c) $\# 2-2$

图 $14 \mathrm{Ti}_{2} \mathrm{AlNb}$ 合金焊接接头 $650{ }^{\circ} \mathrm{C}$ 高温拉伸断口形貌

\section{3 结论}

(1) 试验用固溶态 $\mathrm{Ti}_{2} \mathrm{AlNb}$ 合金母材由 $\alpha_{2}$ 相、 $\mathrm{B} 2$ 相及 $\mathrm{O}$ 相构成的三相合金, 固溶 + 时效态的 $\mathrm{Ti}_{2} \mathrm{AlNb}$ 合金母材由 $\mathrm{B} 2+\mathrm{O}$ 两相组成, 两种热处理 状态的母材均得到了融合良好的焊接接头, 表明 $\mathrm{Ti}_{2} \mathrm{AlNb}$ 合金的惯性摩擦焊接性尚可。

(2) 固溶态及固溶+单时效态的母材焊接接头 焊缝区及热力影响区金相组织基本为 $\mathrm{B} 2$ 相; 焊后 $990{ }^{\circ} \mathrm{C} \times 2 \mathrm{~h}$ 空冷 $+790{ }^{\circ} \mathrm{C} \times 24 \mathrm{~h}$ 空冷双时效态的接头 焊缝区及热力影响区更多的 B2 相转变成晶界网状 $\mathrm{O}$ 相十晶内针状及块状 $\mathrm{O}$ 相组织。焊后 $790{ }^{\circ} \mathrm{C} \times 24 \mathrm{~h}$ 空冷单时效接头焊缝区及热力影响区晶界基本无 $\mathrm{O}$ 相组织存在, B2 相晶内为细密层片状 B2 $+\mathrm{O}$ 相共析 组织。

(3) Ti-20Al-25Nb 型 $\mathrm{Ti}_{2} \mathrm{AlNb}$ 合金惯性摩擦焊接 头室温拉伸试样中, 焊前固溶+焊后双时效处理的接 头抗拉强度最高为 $1193 \mathrm{MPa}$, 且焊后双时效处理 的接头拉伸断口以准解理断裂为主, 而焊后单时效 处理的接头拉伸断口以解理断裂为主, 且解理小平 面尺寸较大, 抗拉强度稍低, 可能与时效处理温度 有关。

(4) 焊前固溶+焊后双时效处理的接头 $650{ }^{\circ} \mathrm{C}$ 高温抗拉强度最高为 $986 \mathrm{MPa}$, 断口上能够观察到
较浅的韧窝, 且为沿晶的韧性断裂, 而焊前双时效 处理的接头断口形貌基本相同，接头断裂形式以解 理断裂为主, 并含有少量的准解理断口。

\section{参 考 文 献}

[1] 冯艾寒, 李渤渤, 沈军. $\mathrm{Ti}_{2} \mathrm{AlNb}$ 基合金的研究进展 $[\mathrm{J}$. 材料与冶金学报，2011，10(1)：30-38.

FENG Aihan, LI Bobo, SHEN Jun. The development of $\mathrm{Ti}_{2} \mathrm{AlNb}$ alloy[J]. Journal of Materials and Metallurgy, 2011, 10(1): 30-38.

[2] 吴爱萍, 邹贵生, 任家烈. $\mathrm{Ti}_{3} \mathrm{Al}$ 合金的发展现状及其连 接技术[J]. 航空制造技术，2007(6): 30-35.

WU Aiping, ZOU Guisheng, REN Jialie. Development and joint technology of Ti3Al alloy[J]. Aeronautical Manufacturing Technology, 2007(6): 30-35.

[3] 乌彦全, 周军, 张春波, 等. Ti-Al 系金属间化合物焊接 技术研究现状[J]. 电焊机, 2019, 49(5): 32-38, 42. WU Yanquan, ZHOU Jun, ZHANG Chunbo, et al. Research status of welding technology for Ti-Al intermetallic compound[J]. Electric Welding Machine, 2019, 49(5): $32-38,42$.

[4] 张建伟, 梁晓波, 程云君, 等. 航空发动机用 $\mathrm{Ti}_{3} \mathrm{Al}$ 合 金和 $\mathrm{Ti}_{2} \mathrm{AlNb}$ 合金研制进展 [J]. 钢铁研究学报, 2011(s2): 545-548.

ZHANG Jianwei, LIANG Xiaobo, CHENG Yunjun, et al. Research and application of $\mathrm{Ti}_{3} \mathrm{Al}$ and $\mathrm{Ti}_{2} \mathrm{AlNb}$ alloys on aero-engine[J]. Journal of Iron and Steel Research, 2011(s2): 545-548.

[5] CHEN B, XIONG H, SUN B, et al. Microstructure evolution and tensile properties of $\mathrm{Ti}_{3} \mathrm{Al} / \mathrm{Ni}$-based superalloy welded joint[]]. Journal of Materials Science and Technology, 2014, 30(7): 715-721.

[6] CHEN B, XIONG H, SUN B, et al. Microstructures and mechanical properties of Ti3 Al/Ni-based superalloy joints arc welded with $\mathrm{Ti}-\mathrm{Nb}$ and $\mathrm{Ti}-\mathrm{Ni}-\mathrm{Nb}$ filler alloys[J]. Progress in Natural Science: Materials International, 2014, 24(4): 313-320.

[7] REN H S, WU X, CHEN B, et al. Microstructures and mechanical properties of Ti3Al/Ni-based superalloy joints diffusion bonded with $\mathrm{Ni}$ and TiNiNb foils[J]. Welding in the World, 2017, 61(2): 357-381.

[8] SHINODA T, ITO K, HAYASHI C. Friction welding of TiAl intermetallic compound[J]. Welding International, 1997, 11(3): 200-205.

[9] XU Q, CHATURVEDI M C, RICHARDS N L. The role of phase transformation in electron-beam welding of TiAl-based alloys[J]. Metallurgical \& Materials Transactions A, 1999, 30(7): 1717-1726.

[10] DONG H G, YU L Z, GAO H M, et al. Microstructure 
and mechanical properties of friction welds between TiAl alloy and $40 \mathrm{Cr}$ steel rods[J]. Transactions of Nonferrous Metals Society of China, 2014, 24(10): 3126-3133.

[11] DONG H G, YU L Z, GAO H M, et al. Direct friction welding of TiAl alloy to $42 \mathrm{CrMo}$ steel rods[J]. Materials and Manufacturing Processes, 2015, 30(9): 1104-1108.

[12] CHEN Y, ZHANG K, HU X, et al. Study on laser welding of a Ti-22Al-25Nb alloy: microstructural evolution and high temperature brittle behavior[J]. Journal of Alloys \& Compounds, 2016, 681: 175-185.

[13] PALANIVEL R, DINAHARAN I, LAUBSCHER R F. Assessment of microstructure and tensile behavior of continuous drive friction welded titanium tubes[J]. Materials Science \& Engineering A, 2017, 687: 249-258.

[14] 谭立军, 姚泽坤, 周伟, 等. Ti-22Al-25Nb 与 TC11 异 种钛合金的线性摩擦焊接 [J]. 塑性工程学报, 2009, 16(6): 135-138.

TAN Lijun, YAO Zekun, ZHOU Wei, et al. Linear friction welding of dissimilar titanium alloys $\mathrm{Ti}-22 \mathrm{Al}-25 \mathrm{Nb}$ and TC11[J]. Journal of Plasticity Engineering, 2009, 16(6): 135-138.

[15] CHEN X, XIE F Q, MA T J, et al. Microstructure evolution and mechanical properties of linear friction welded Ti2AlNb alloy[J]. Journal of Alloys \& Compounds, 2015, 646: 490-496.

[16] CHEN X, XIE F Q, MA T J, et al. Microstructure evolution and mechanical properties of linear friction welded $\mathrm{Ti}_{2} \mathrm{AlNb}$ joint during solution and aging treatment[J]. Materials Science \& Engineering A, 2016, 668: 125-136.

[17] 贺建超, 张田仓, 李菊. 热处理对 $\mathrm{Ti}_{2} \mathrm{AlNb}$ 线性摩擦焊 接头组织与硬度的影响 [J]. 焊接学报, 2019, 40(4):

119-124, 166.

HE Jianchao, ZHANG Tiancang, LI Ju. Effect of heat treatment on microstructure and hardness of $\mathrm{Ti}_{2} \mathrm{AlNb}$ linear friction welding joint $[\mathrm{J}]$. Transactions of the China Welding in Institution，2019，40(4): 119-124， 166.

[18] 周军，乌彦全，张春波，等. $\mathrm{Ti}_{2} \mathrm{AlNb}$ 金属间化合物惯 性摩擦焊接头组织及性能分析 $[\mathrm{J}]$. 焊接学报, 2018, 39(11): 27-32, 130 .

ZHOU Jun, WU Yanquan, ZHANG Chunbo, et al. Analysis of microstructures and properties of $\mathrm{Ti}_{2} \mathrm{AlNb}$ intermetallic compound inertia friction welded joints [J]. Transactions of the China Welding in Institution, 2018, 39(11): 27-32, 130 .

[19] WANG W, ZENG W, XUE C, et al. Quantitative analysis of the effect of heat treatment on microstructural evolution and microhardness of an isothermally forged Ti-22Al-25Nb(at.\%) orthorhombic alloy[J]. Intermetallics， 2014，45: 29-37.

作者简介: 周军, 男, 1963 年出生, 硕士, 研究员, 博士研究生导师。 主要研究方向为摩擦焊接工艺及装备。

E-mail: mch_zhoujun@126.com 